\title{
Stabilization of Mineral Precursors by Intrinsically Disordered Proteins
}

\author{
Ashit Rao, Markus Drechsler, Stefan Schiller, Martin Scheffner, Denis Gebauer, \\ and Helmut Cölfen*
}

Biogenic nucleation and crystallization occur in confined spaces with defined interfacial properties. However, the regulatory functions of organic players in the stabilization and transport of inorganic precursors such as ion clusters, liquid-condensed phases, and amorphous particles are unclear. Given the prevalence of unstructured proteins in biogenic materials, the present study investigates the effects of biomineral-associated, intrinsically disordered protein domains with simple and repetitive amino acid compositions on mineral nucleation and their capability to form distinct supramolecular assemblies. The quantitative assessment and structural evaluation of the nucleation process reveal that disordered regions confine hydrated mineral precursors within vesicles, transiently suppressing mineral precipitation. Stabilization of the amorphous mineral is attributed to protein self-association and restructuration toward $\beta$-configurations, triggered by specific bioinorganic interactions. In consequence, the conditioned macromolecules localize at phase boundaries formed upon liquid-liquid demixing of mineral precursors and stabilize the fluidic mineral precursors against crystallization. Thus, the conformational plasticity and self-association of intrinsically disordered sequences in response to crystallization environments mediates the selection of functional macromolecular subensembles dedicated to biomaterial growth.

distinct category referred to "natively denatured," "natively unfolded," or "intrinsically disordered/ unstructured proteins" (IDPs). Due to their intrinsic pliability as well as sequence characteristics of low complexity and amino acid compositional bias, ${ }^{[2]}$ IDPs exhibit fascinating modes of function including induced-folding, ${ }^{[3]}$ conformational adaptability toward binding sites, ${ }^{[4]}$ and a unique phase behavior as hydrogels or liquid-like droplets. ${ }^{[1,5]}$ Given the recent elucidation of the contributions of IDPs in cellular homeostasis, their roles in the formation and maintenance of biomaterials also require attention.

The prevalence of IDPs in the extracellular milieu is high relative to the complete cellular proteome, ${ }^{[6]}$ reflecting the participation of intrinsically disordered regions (IDRs) in conditioning soft and hard extracellular matrices. The related functions of IDRs appear to encompass cell-matrix adhesion, with possible contributions in the organization and maintenance of the matrix structure. ${ }^{[6]}$ Although

\section{Introduction}

Previous notions about biomolecular interactions assumed absolute structural complementarity between well-folded partners. Subsequent advances in analytical and computational methods revealed that bioactive domains do not consistently exhibit defined, rigid conformations under native conditions. ${ }^{[1]}$ These observations have induced paradigm shifts in the understanding of protein structure and function, establishing a several biomineral-associated proteins exhibit high propensities of structural disorder such as amelogenin from enamel tissue, ${ }^{[7]}$ bone-associated sialoprotein and osteopontin, ${ }^{[8]}$ SpSM50 from sea urchin spines, ${ }^{[9]}$ Sillafins regulating biosilicification, ${ }^{[10]}$ Starmarker from fish otoliths, ${ }^{[11]}$ as well as AP7, Asprich, n16, PFMG1, and Shematrin from shell materials, ${ }^{[12]}$ the molecular functions of IDPs in guiding mineral nucleation and crystallization are not clear. Few recent studies indicate the potential contributions of these unstructured polymers in biomineralization
Dr. A. Rao, Dr. D. Gebauer, Prof. H. Cölfen

Physical Chemistry

Department of Chemistry

University of Konstanz

Konstanz 78464, Germany

E-mail: Helmut.Coelfen@uni-konstanz.de

Dr. A. Rao, Dr. S. Schiller

Freiburg Institute for Advanced Studies

and Centre for Biosystem Analysis

Albert-Ludwigs-Universität Freiburg

Freiburg 79104, Germany

\author{
Dr. A. Rao \\ Faculty of Science and Technology \\ Physics of Complex Fluids \\ University of Twente \\ PO Box 217 Enschede 7500 AE, The Netherlands \\ Dr. M. Drechsler \\ Bavarian Polymer Institute \\ Keylab: Electron and Optical Microscopy \\ University of Bayreuth \\ Bayreuth 95440, Germany \\ Prof. M. Scheffner \\ Department of Biology \\ University of Konstanz \\ Konstanz 78464, Germany
}


such as (i) the assembly of protein monomers into supramolecular structures that organize the mineral phase over multiple length scales, ${ }^{[12 \mathrm{~d}]}$ (ii) crowding-induced modulation of mineralization reactions and confinement-mediated transient stabilization of mineral precursors within pores of organic networks, ${ }^{[13]}$ (iii) phase separation of liquid- or gel-like organic phases that modulate mineralization, ${ }^{[14]}$ (iv) mechanical reinforcement of hybrid materials via inter- and intracrystalline occlusion of bioelastomers, ${ }^{[15]}$ and also (v) prospective regulatory checkpoints arising from a predisposition of redundant sequences toward effective post-translational alterations. ${ }^{[16]}$ Consistent with these putative functions, spine- and nacre-associated IDPs appear to not only regulate mineral structure, but also modulate the formation and stability of crystal precursors..$^{[9,12 a, 17]}$ Accompanying variations in the self-association propensity and conformational flexibility of IDPs are related to specific interactions with ion species and transient mineral phases. For example, mineral precursors induce disorder-to-order structural transitions in proteins, which might subsequently form gels with capacities for distinct ionic interactions. ${ }^{[18]}$ In all, these studies suggest that mineral nucleation and crystallization might favor a set of macromolecular conformations from the entire accessible ensemble, conducive for regulating concomitant mineralization events. This notion is also supported by the conformational bias of unstructured domains toward globular or extended states, influenced by the charge content of polypeptide backbones and prevalent ionic conditions. ${ }^{[19]}$ Hence, it is vital to understand the dynamics of unstructured biopolymers in response to inorganic species in form of solutes, fluidic precursors, and nanoparticles for elucidating the molecular mechanisms of the formation, growth, and maintenance for biomaterials.

Considering the sea urchin spicule proteome, ${ }^{[20]}$ bioinformatics analyses reveal the distribution of structural disorder and low sequence complexity (Figure S1, Supporting Information). Of about 200 proteins, a significant proportion contain disordered regions as well as repetitive and compositionally simple low complexity (LC) sequences, for which the biochemical functions in the nucleation and crystallization of inorganic phases are uncertain. To decipher the exact roles of LC-IDPs in biomineralization, in this study, recombinant biomineralassociated LC unstructured sequences are investigated for their effects on the nucleation and crystallization of calcium carbonate $\left(\mathrm{CaCO}_{3}\right)$ phases. On account of their indispensable roles in sea urchin skeletogenesis, LC-IDRs of SpSM50 from Strongylocentrotus purpuratus and its ortholog LSM34 from Lytechinus pictus in spiculogenesis are selected. [21] The third additive is derived from a mesenchyme cell-surface protein, MSP130 which is implicated in calcium uptake and deposition. ${ }^{[22]}$ An unstructured region from Prisilkin-39 from the prismatic layer of Pinctada fucata is also investigated because of a distinct enrichment of Tyr and Ser residues as well as putative functions in crystallization and scaffold construction. ${ }^{[23]}$ To overcome the poor expression and low solubility of the recombinant proteins, a N-terminal small ubiquitin-like modifier (SUMO) tag is utilized. ${ }^{[24]}$ To address the contributions of the fusion tag toward mineralization, its p53TAD fusion product is applied as a reference (Figure S2, Supporting Information). Together the effects of these domains on $\mathrm{CaCO}_{3}$ nucleation are quantitatively evaluated under defined solution conditions by

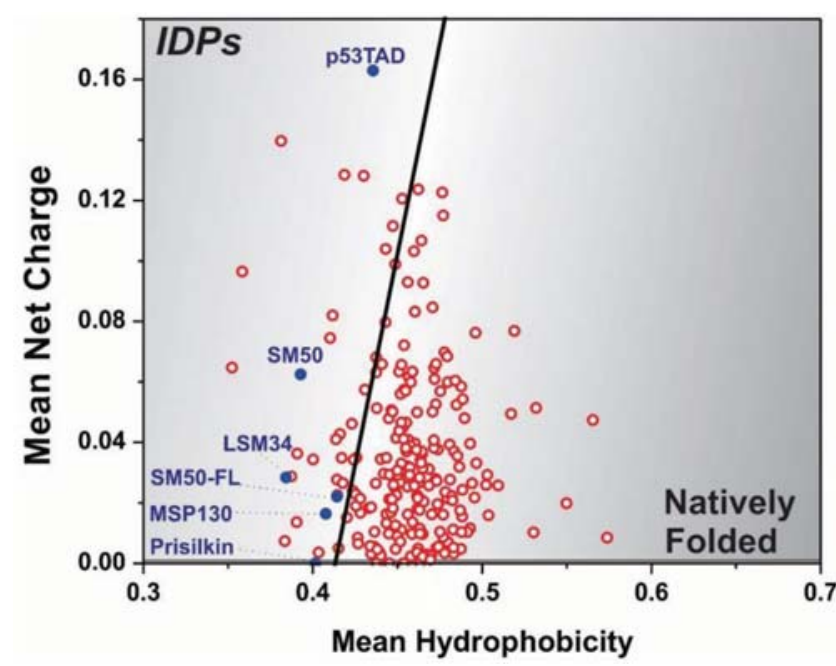

Figure 1. Amino acid composition derived charge-hydropathy plot of the protein additives (blue dots) in relation to the sea urchin spicule proteome (empty circles). The line represents the delineation of unstructured and natively folded proteins. ${ }^{[26]}$ In addition to sequences of the investigated proteins (Figure S4, Supporting Information), the full length SM50 (SM50-FL) is indicated.

applying a potentiometric titration-based methodology. ${ }^{[25]}$ Mineralization-related structural transitions are investigated in the solution state by analytical ultracentrifugation (AUC), dynamic light scattering (DLS), and cryogenic-transmission electron microscopy (cryo-TEM).

\section{Results and Discussions}

\subsection{Bioinformatics Evaluation}

For an overview of sequence parameters and disorder propensities, the charge-hydropathy $(\mathrm{CH})$ distribution of the LC-IDPs is depicted in relation to the entire sea urchin spicule proteome (Figure 1). The $\mathrm{CH}$ distribution provides an empirical distinction of globular and unstructured protein. Members of the spicule proteome cluster at the zone delineating unstructured and globular proteins. ${ }^{[26]}$ This localization reflects intermediate levels of structural pliability, subject to in vivo conditions of post-translational modifications, ionic fluxes, $\mathrm{pH}$, and crowding. The LC-IDRs show transitional $\mathrm{CH}$ values, with similar hydrophobicities but varying values of net charge (Figure 1). Prisilkin-39 has a relative low net charge because of a sequence bias favoring Ser and Try (Figure S2, Supporting Information). On the other hand, the spicule-associated LC-IDRs are enriched in Gln residues and present higher net charge values. This is also indicated by the respective isoelectric points and amino acid compositions (Table S1, Supporting Information). Thus, LC-IDRs from SM50 and LSM34 are more charged and unstructured in comparison to the MSP130 and Prisilkin-39 regions. Significant disorder propensities of the IDRs are confirmed by applying DISOPRED, IUPRED, and RONN algorithms (Figure S3, Supporting Information). Minor disparities are observed between different prediction tools because of distinct evaluation algorithms with DISOPRED 
involving crystal structure information based sequence classification, ${ }^{[27]}$ IUPRED based on total pairwise inter-residue interaction energy, ${ }^{[28]}$ and RONN applying a neural network pattern recognition algorithm using datasets of X-ray crystallography and NMR structures. ${ }^{[29]}$ Interestingly, higher propensities of structural disorder are presented by biomineral-associated protein sequences enriched in basic amino acids (Figure S3, Supporting Information). From the $\mathrm{CH}$ plot (Figure 1), the fulllength SM50 is less unstructured in comparison to its constitutive glycine rich domain, which suggests a folded N-terminal C-type lectin domain. ${ }^{[9]}$

\subsection{Quantitative Assessment of Mineral Nucleation}

The effects of recombinant IDRs on mineral nucleation are examined (Figures S4 and S5, Supporting Information). The developments of free $\mathrm{Ca}^{2+}$ ions and base counter-titration enable the quantitative comparison of the time required for mineral precipitation, ion-association in the prenucleation regime, and the nature of initially nucleated mineral phases (Figures 2 and 3). ${ }^{[25 b]}$ The concentration- and $\mathrm{pH}$-dependent nucleation trends are discussed in terms of a mean normalized parameter $\left(F_{0}\right)$, defined as the quotient of the nucleation time points of additive containing and reference experiments. ${ }^{[30]}$ At $\mathrm{pH}$ 9.75, the LC-IDR of Prisilkin-39 is the most potent nucleation inhibitor $\left(F_{0}=4\right)$. On the other hand, at $\mathrm{pH} 9.0$, the unstructured domains of SM50 and LSM34 are better inhibitors of mineral nucleation with corresponding $F_{0}$ values of 3.1 and 2.3 (Figure 2). Taking into account the simulated $\mathrm{pH}$-related speciation (Figure S6 and Table S1, Supporting Information), p53TAD is expected to significantly impede nucleation at lower $\mathrm{pH}$ in assumption of predominant ion-complexation. However, as experimentally determined, the LC-IDRs are better inhibitors of mineral precipitation (Figure 3). Since these recombinant LC-IDRs are devoid of acidic residues such as Asp and

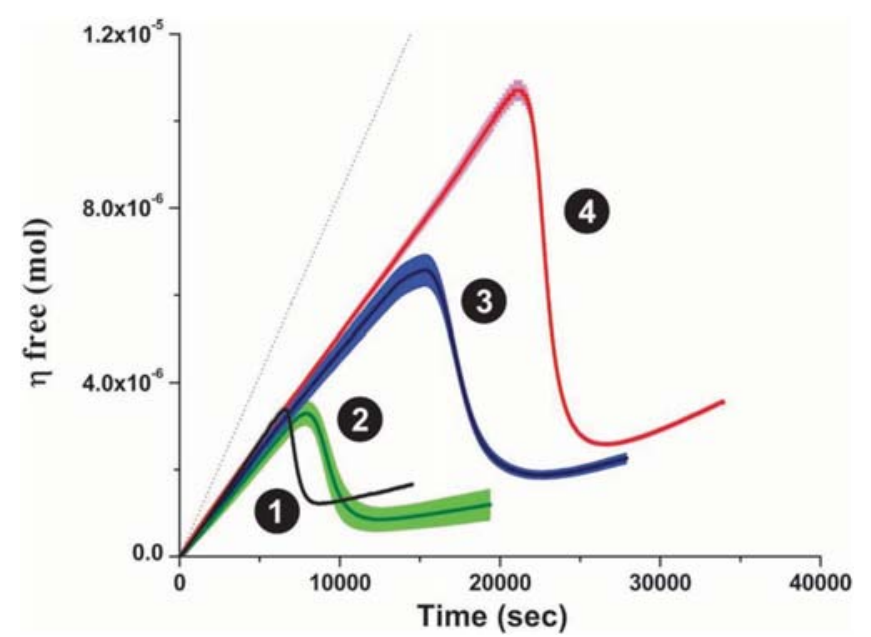

Figure 2. Time development of free $\mathrm{Ca}^{2+}$ ions at $\mathrm{pH} 9.0$ during mineral nucleation (1) in the absence of additives (black) and in the presence of fusion products based on IDRs from (2) Prisilkin-39 (green), (3) LSM34 (blue), and (4) SM50 (red). Shaded zones represent \pm 1 standard deviation. The added $\mathrm{Ca}^{2+}$ ion content is represented by the dotted line.
Glu which effectively inhibit calcification ${ }^{[31]}$ as well as posttranslational modifications such as glycosylation and phosphorylation, which influence self-association tendencies, ${ }^{[32]}$ the delayed onset of mineral nucleation in the presence of highly basic LC-IDRs likely has alternative origins based on the conformational and colloidal stabilization of mineral precursors and associated dynamics of self-association and structure of the disordered biopolymers. As the inhibited nucleation might not affect the locus of the binodal limit for liquid-liquid demixing of the aqueous calcium carbonate solutions, ${ }^{[33]}$ an extended lifetime of liquid precursors can be advantageous for an effective mineralization of the nanospaces within complex organic scaffolds. As depicted by the effects of protein concentrations on mineral nucleation trends at $\mathrm{pH} 9.0$ and 9.75 (Figures S4 and S5, Supporting Information), the supersaturation contents required for mineral nucleation are significantly affected by the applied contents of macromolecules. This reflects the contributions of local crowding and confinement in stabilizing fluidic mineral precursors, representing a vital bioinorganic checkpoint regulating mineral form and growth. ${ }^{[34]}$

From the prenucleation regimes, significant equilibrium shifts toward ion-association mediated by Prisilkin-39 and MSP130 derivatives are seen (Figure 3). These observations are not due to strong ion complexation by the protein species, which otherwise elicit initial offsets in the development of free $\mathrm{Ca}^{2+}$ ions. ${ }^{[25 b]}$ The solubility products of initially nucleated mineral phases are also influenced by the LC-IDPs. At pH 9.0, LCIDRs of SM50, LSM34, and MSP130 lead to increased solubility products relative to the neat buffer experiments (Figure 3). Since the observed solubility products of the nucleated phase are high relative to those of amorphous $\mathrm{CaCO}_{3}$ particles, ${ }^{[25 b]}$ a precursor mineral phase is transiently stabilized by the LCIDRs. Polarization microscopy of corresponding samples confirms the stabilization of an amorphous, liquid-like phase (Figure S7, Supporting Information). The amorphous droplets are indicative of either coacervate formation via ionic interactions or a (bio)polymer-stabilized liquid precursor of $\mathrm{CaCO}_{3} \cdot{ }^{[35]}$ While dense liquid phases do not exhibit solubilities, the microscopic observations indicate that the IDRs inhibit dehydration and the higher solubility threshold in presence of the macromolecules is due to an increased water content in the intermediate ACCs when compared to the reference scenario. As an exception among the LC-IDPs, Prisilkin-39 leads to lower solubility products corresponding to vaterite, a crystalline $\mathrm{CaCO}_{3}$ polymorph at higher $\mathrm{pH}$ (Figure 3F). Thus, Prisilkin-39 might promote the kinetic destabilization of the liquid-like, and subsequently solid mineral precursors toward crystalline forms. In all, since an inhibition of nucleation prolongs the lifetime of transient fluidic precursors in additive-controlled reactions, ${ }^{[33,36]}$ a retention of the corresponding bioinorganic interactions can extend beyond the point of mineral nucleation and result in the kinetic stabilization of highly hydrated amorphous mineral phases (Figure 3, and Figure S7, Supporting Information).

\subsection{Size and Structure Evolution of Nucleation Species}

To address the progression of organic-inorganic interactions during the nucleation reactions, the time evolution of 

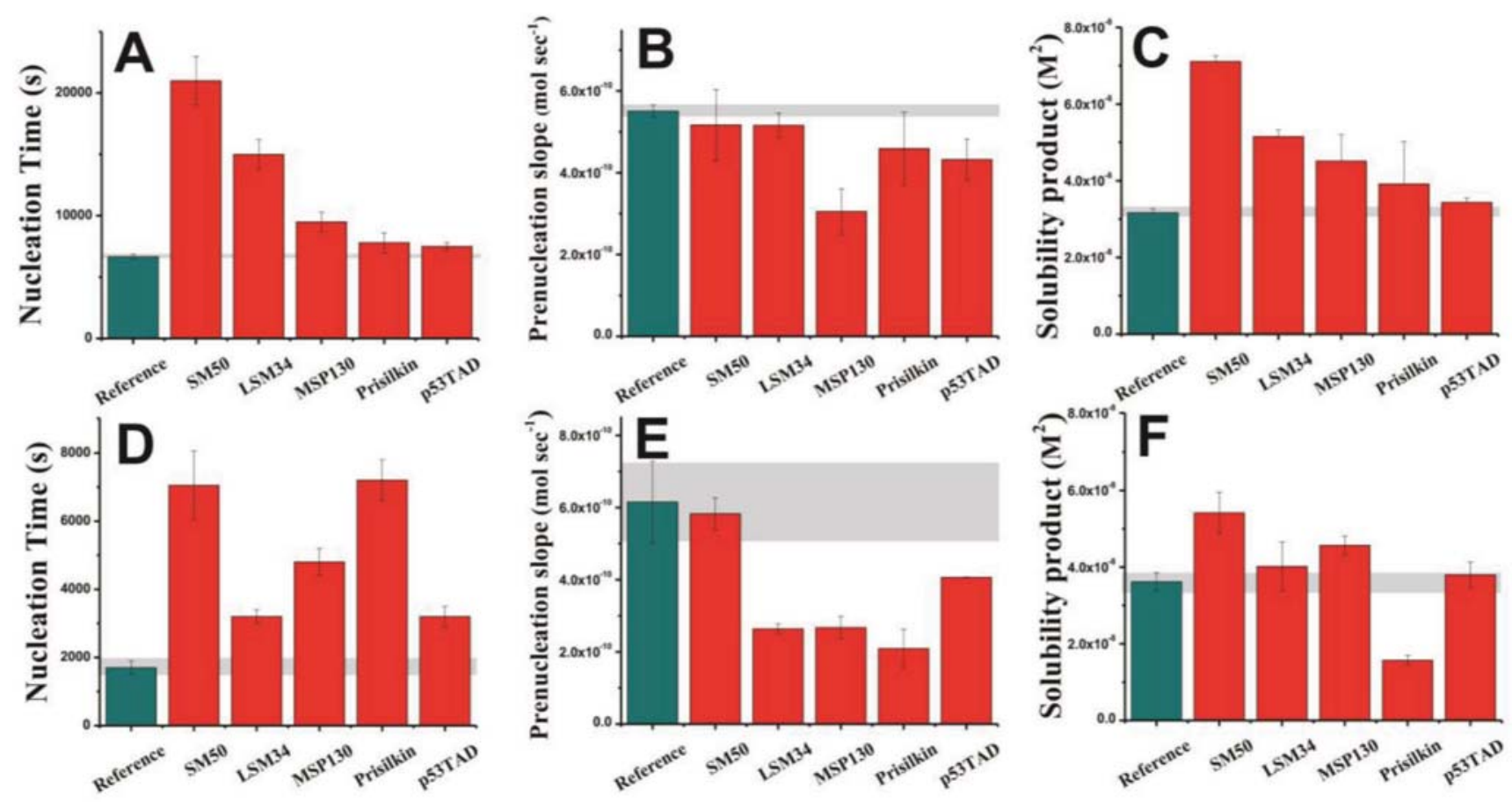

Figure 3. Bar plots representing the early stages of $\mathrm{CaCO}_{3}$ nucleation in terms of the $\left.A, D\right)$ time required for mineral nucleation, $\left.B, E\right)$ ion-association in the prenucleation regime, and $\mathrm{C}, \mathrm{F}$ ) solubility of initially formed phase in reference (green) and protein-containing (red) titrations at (A-C) pH 9.0 and (D-F) $\mathrm{pH}$ 9.75. In reference experiments, the solubility product of the initially nucleated mineral phase corresponds to proto-calcite and proto-vaterite $\mathrm{ACC}$ at $\mathrm{pH} 9.0$ and 9.75 , respectively. Error bars represent \pm 1 standard deviation.

hydrodynamic radii of species is shown (Figure S8, Supporting Information). The DLS-determined particle sizes collectively represent protein species, protein-ion complexes, large ionassociates, and mineral particles. During early prenucleation stages, the particle sizes range between 0.8 and $4.0 \mathrm{~nm}$. Following this stage until nucleation, the particle sizes exhibit temporal developments, suggestive of protein associates and early inorganic forms such as aggregating ion clusters and emergent liquid condensed phases. ${ }^{[33,37]}$ Interestingly, in the postnucleation regimes under regulation of the LC-IDRs from SM50, LSM34, and MSP130, the particle sizes range between $420 \pm 200$ and $790 \pm 320 \mathrm{~nm}$. On the other hand, in the presence of LC-IDRs from Prisilkin-39 and p53TAD, the mean particle sizes are higher, in the range of $1350 \pm 350$ and $1800 \pm 700 \mathrm{~nm}$ consistent with reference values. The assembly or aggregation of proteins often occurs near their isoelectric point. In this scenario, the IDRs with pIs close to 9.0 (i.e., Prisilkin-39 and MSP130 based) should exhibit greater selfassociation. However, from the DLS experiments (Figure S8, Supporting Information), the p53TAD derivative forms larger particles during mineral nucleation, relative to MSP130. Thus, it appears that the relation between self-association and isoelectric point may only partially hold for the investigated domains. To understand these distinct time developments of the hydrodynamic radii, the protein-mineral interactions are further characterized using high-resolution techniques.

Nanoscopic structures formed in course of mineral nucleation are imaged under cryogenic conditions (Figure 4). In the nascent stages of nucleation $(F=0.2)$, that is, prior to the liquidliquid locus, ${ }^{[33]}$ the LC-IDRs are more prone to self-association in comparison to the reference p53TAD experiments. The LCIDRs form cloud- or sheet-like structures that exhibit a relatively low electron contrast. This is suggestive of protein self-association driven by interactions with ions and ion-associates. Prior to mineral nucleation, the LC-IDRs of SM50, LSM34, MSP130, and Prisilkin-39 undergo a remarkable self-assembly process and mediate vesicle formation (100-300 nm in diameter), not observed in the presence of the SUMO-p53TAD reference. The corresponding diffraction patterns are suggestive of amorphous compositions of the vesicular structures. The absence of vesicles in the initial stages of nucleation verifies that the self-assembly of LC-IDRs specifically occurs in response to increasing supersaturation. Since the formation of vesicles initiates at $F$ values greater than 0.2 (Figure 4), vesicle formation is specifically triggered by liquid-liquid separation reactions. ${ }^{[33]}$ The removal of solvent compromises the vesicle structure and promotes the transformation of the enclosed amorphous phase to crystalline $\mathrm{CaCO}_{3}$ (Figure S9, Supporting Information). Ringlike morphologies with centrally located crystallites suggest considerable volume losses due to an amorphous-to-crystalline phase transition and osmotic expansion of the vesicle walls to a hypotonic environment arising from the low solubility of crystalline $\mathrm{CaCO}_{3}$. In all, the cryo-TEM and titration experiments show that the LC-IDRs specifically concert a confinement-based stabilization of highly hydrated, amorphous mineral precursors. Under cryogenic conditions, an interesting observation is the nucleation of vaterite superstructures in proximity of the vesicles composed of the Prisilkin-39 domain (arrows, Figure S10, Supporting Information). Deformed vesicles in proximity suggest that crystalline superstructures can emerge 


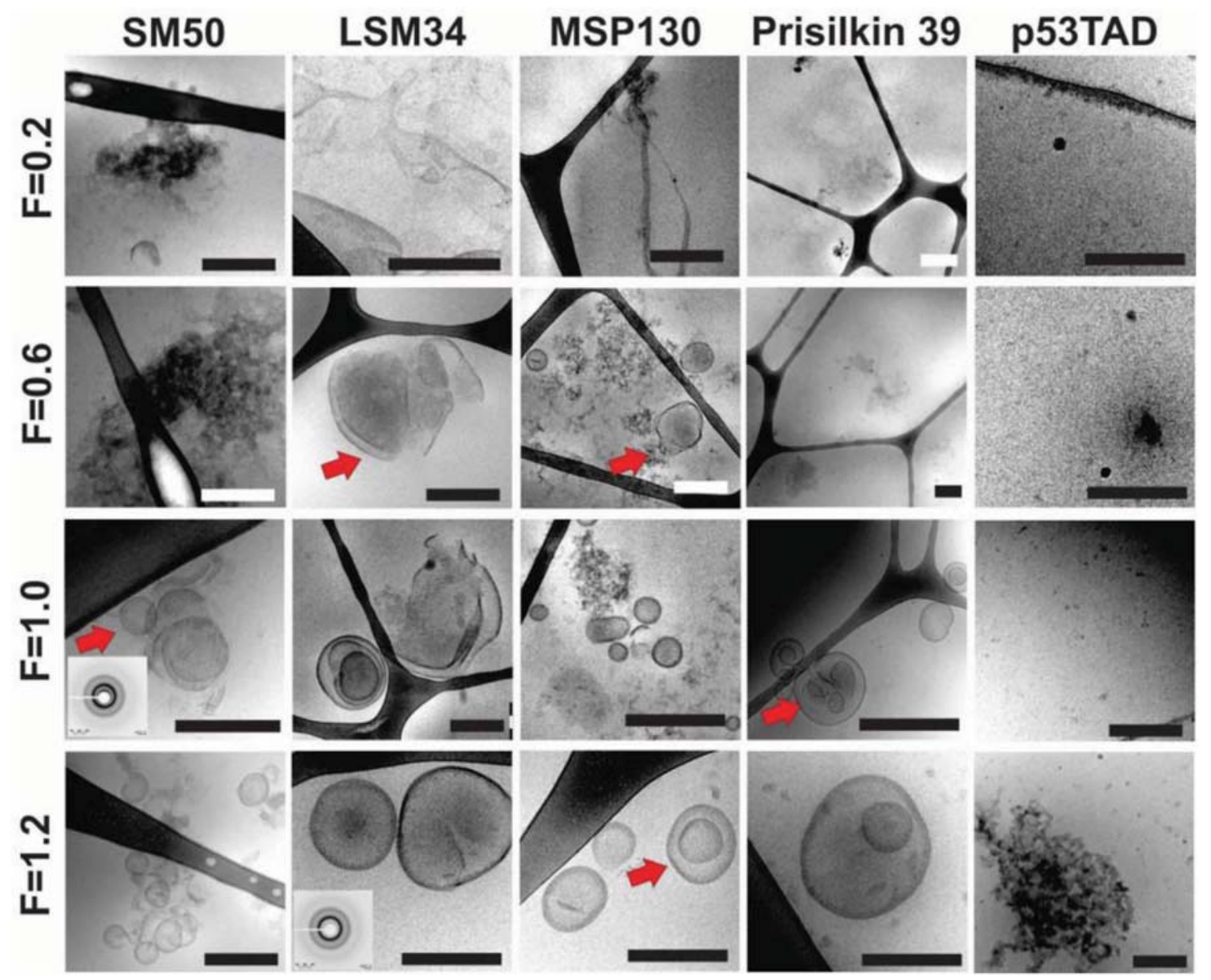

Figure 4. Cryo-TEM images representing structural developments during distinct stages of mineral nucleation in presence of protein additives. For each experiment, the time points are normalized by using the respective nucleation time point, wherein a value of 1 for the scaling factor $(F)$ corresponds to the nucleation event, that is, the drop in free $\mathrm{Ca}^{2+}$ content in the respective titration run. Arrows indicate the presence of vesicles in the temporal regimes between $F$ values of 0.6 and 1.2. Scale bars represent $200 \mathrm{~nm}$.

from the dissolution-precipitation of fluidic mineral precursors transiently stabilized within vesicles, consistent with in vivo studies. ${ }^{[38]}$ However, whether amorphous-to-crystalline transformations occur via solid state or dissolution-precipitation routes is also dependent on applied reaction environments, hydration conditions, as well as the precursor particle size and stability. ${ }^{[39]}$

The relative efficacies of LC-IDP mediated phase stabilization are indicated by the intensity ratio of $v_{2}\left(864 \mathrm{~cm}^{-1}\right)$ and $v_{4}$ $\left(712 \mathrm{~cm}^{-1}\right)$ peaks of amorphous $\mathrm{CaCO}_{3}$ and calcite from Fourier transform infrared (FTIR) spectra ${ }^{[40]}$ (Figure S11, Supporting Information). Mineral products from reference and p53TAD containing reactions provide similar intensity ratios which correspond to baseline values for calcite-rich compositions (Table S2, Supporting Information). On the other hand, mineral phases nucleated in presence of the LC-IDRs contain higher relative contents of amorphous mineral, as evident from higher intensity ratios. In view of the low solubility of the LC-IDRs in carbonate buffer, secondary structure elements are determined from the amide IR bands of the mineralized pellets (Figure S12, Supporting Information). ${ }^{[41]}$ In the amide I region, the spectra of the LC-IDRs display a peak at $1609 \mathrm{~cm}^{-1}$, suggestive of $\beta$ structures typical of either amorphous protein aggregates or amyloid fibrils. The amide II band at $1577 \mathrm{~cm}^{-1}$ is assigned to the $\mathrm{N}-\mathrm{H}$ bending vibration. For further validation, the protein structures are tested with fluorescence and tinctorial assays selective for $\beta$-strand arrangements in amyloid-like fibrils. ${ }^{[42]}$ ThT-treated LC-IDRs aggregates exhibit fluorescence, whereas Congo red produces apple-green birefringence in fibril-like structures and stains larger organic assemblies red (Figure S13, Supporting Information). This confirms the participation of amyloid-like $\beta$ conformations in the mineralization-induced self-assembly of the LC-IDRs. Simulating the mineralizationrelated self-association of the IDRs, fluorospectrometric assays are performed using ThT at different arginine concentrations (Figure S12, Supporting Information). Since arginine is a protein-solubilizing agent, it produces distinct contents of soluble protein fractions in a concentration dependency. ${ }^{[33]}$ The emission signal of ThT-treated LC-IDRs from SM50, LSM34, Prisilkin-39, and MSP130 decrease in proportion to the applied contents of Arg, an interprotein interaction disrupting cosolute. ${ }^{[43]}$ At high Arg contents $\left(200 \times 10^{-6} \mathrm{M}\right)$, the fluorescence emission intensities at $490 \mathrm{~nm}$ decrease by 72, 72, 38, and 45\% for SM50, LSM34, Prisilkin-39, and MSP130, respectively, in comparison to corresponding values in the absence 
of enhanced protein solubility. Thus, the investigated LC-IDRs appear biochemically tuned to undergo disorder-to-order structural transitions favoring amyloid-like $\beta$ contents in solution environments that suppress protein solubility.

To characterize transitional species involved in the process of protein self-association and organization, the distributions of frictional ratio $\left(f / f_{0}\right)$ of the SM50-derived LC-IDR are accessed by the 2D spectra (2DSA) analyses of AUC sedimentation velocity data $^{[64]}$ (Figure S14, Supporting Information). This enables the computational determination of the size and shape distributions of sedimenting species. In addition to a collapsed globular monomeric species (with a $f / f_{0}$ value of 1.1 and an $a$ value of $2.1 \mathrm{~nm})$, relatively large oligomers $(120,240$, and $840 \mathrm{kDa})$ are identified with corresponding $f / f_{0}$ values of $1.6,1.7$, and 3.6, respectively. The oligomers between 120 and $240 \mathrm{kDa}$ correspond to axial ratio of 12.3 and 15.3 , respectively, in assumption of prolate models. While the small oligomers have an extended shape, the larger species are highly asymmetric, possibly sheetlike. This self-association behavior is also verified by TEM analyses of the protein samples in insoluble states and in Arginduced partially solubilized forms (Figure S14, Supporting Information), suggesting the association of fibril-like elements into sheets in course of self-assembly. Thereby, a strong indication emerges for the engulfment of transient fluidic mineral precursors by the accumulation or the nucleation and growth of sheet-like organic assemblies at the liquid-liquid interfaces, realizing the vesicular structures. For instance, in the prenucleation regime, ionic interactions induce the formation of sheetlike structures and fibrils for LSM34- and MSP130-based IDRs (Figure 4). In this regard, the molecular cosolute entities and mediators of protein solubility related to biogenic nucleation and crystallization emerge crucial in the selection of conformational subensembles of macromolecules suited for generating functional inorganic-organic interfaces.

Recent progress in the understanding of nucleation and crystallization phenomena show that metastable fluidic mineral precursors can be transiently stabilized by additives. ${ }^{[33,36,44]}$ In the present study, the essential roles of interfaces between liquid-like mineral precursors and the bulk solvent contributing to a confinement-based stabilization are indicated by (i) the primary sequences of LC-IDR lacking strong amphiphilicity (Figure S2, Supporting Information) and also (ii) the induction of vesicle formation prior to mineral nucleation (Figure 4), subsequent to the liquid-liquid binodal demixing. ${ }^{[33]}$ These observations hint that the interfacial area associated with highly hydrated, mineral nanodroplets govern protein self-association and the construction of vesicle walls. In order to reproduce a transient liquid-liquid interface and its effects on the selfassembly of LC-IDRs, aqueous solutions of rhodamine-labeled LC-IDRs are treated with tetrahydrofuran (THF). With a low dielectric constant $\left(7.58\right.$ at $\left.25^{\circ} \mathrm{C}\right)$, THF is applied as an antisolvent, substituting the effect of mineral precursors. By using fluorescence microscopy, spontaneous vesicle formation is observed after the addition of THF to aqueous solutions of the LC-IDRs (arrows, Figure 5). Thus, the preferential accumulation of the LC-IDRs at liquid-liquid phase boundaries can mediate vesicle formation. The consequences of these hybrid structures are not limited to a confinement-based phase stabilization of mineral precursors as demonstrated here, but can
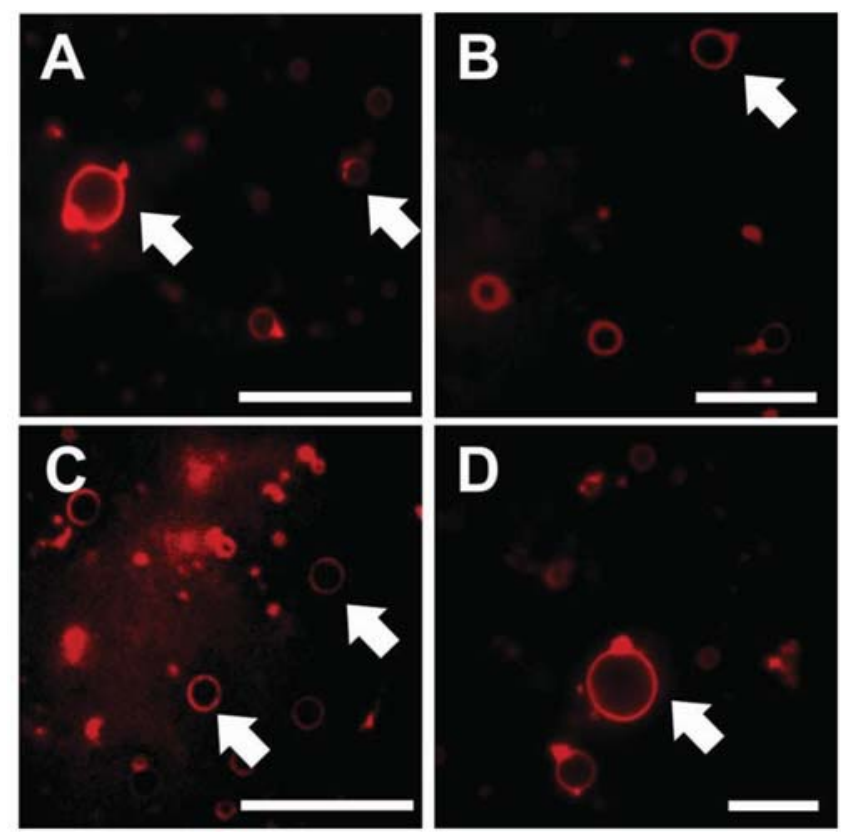

Figure 5. Fluorescence microscopy images of vesicles composed of rhodamine labeled SUMO fusion proteins containing LC-IDRs of A) SM50, B) LSM34, C) MSP130, and D) Prisilkin-39. Scale bar represents $200 \mu \mathrm{m}$.

also subsequently generate complex mineral superstructures (Figure S10, Supporting Information) and hierarchical composites such as bone tissue. ${ }^{[35 a]}$

\subsection{Mechanistic Outlook}

Vesicular structures transporting amorphous mineral are observed in several biomineral systems. For instance, the packaging of mineral precursors in vesicular or vacuole-like structures is reported in nacre material, ${ }^{[45]}$ fin bony rays of zebrafish, ${ }^{[46]}$ mammalian bone tissue, ${ }^{[47]}$ the coccolithophore, Emiliania huxleyi ${ }^{[48]}$ and primary mesenchymal cells regulating sea urchin spiculogenesis. ${ }^{[38]}$ Thus, the stabilization of mineral precursors in vesicle-based confined volumes is a widespread biological strategy for regulating material formation. This study shows that highly basic, unstructured biomineralization protein domains can effectively stabilize liquid-like mineral precursors against crystallization via a confinement-based mechanism. Considering the participation of LC-IDR-based vesicles in stabilizing fluidic mineral precursors, the proposed mechanism draws from the bidirectional influences of organic and inorganic players during nucleation and crystallization reactions, spanning beyond bioinorganic interactions solely based on chemical functionality (Figure 6). Prior to mineral nucleation, ions and ion clusters lower the solubility of LC-IDRs and thereby condition the unstructured regions toward self-association and conformational transitions involving disorder-to-order reconfigurations favoring $\beta$ structures. Concomitantly, these organic-inorganic interactions modulate ion-ion association, affect the stability of PNCs, and also determine the nucleation point with a tendency toward inhibiting mineral precipitation. The distinct protein-dependent nucleation trends (Figure 3) 


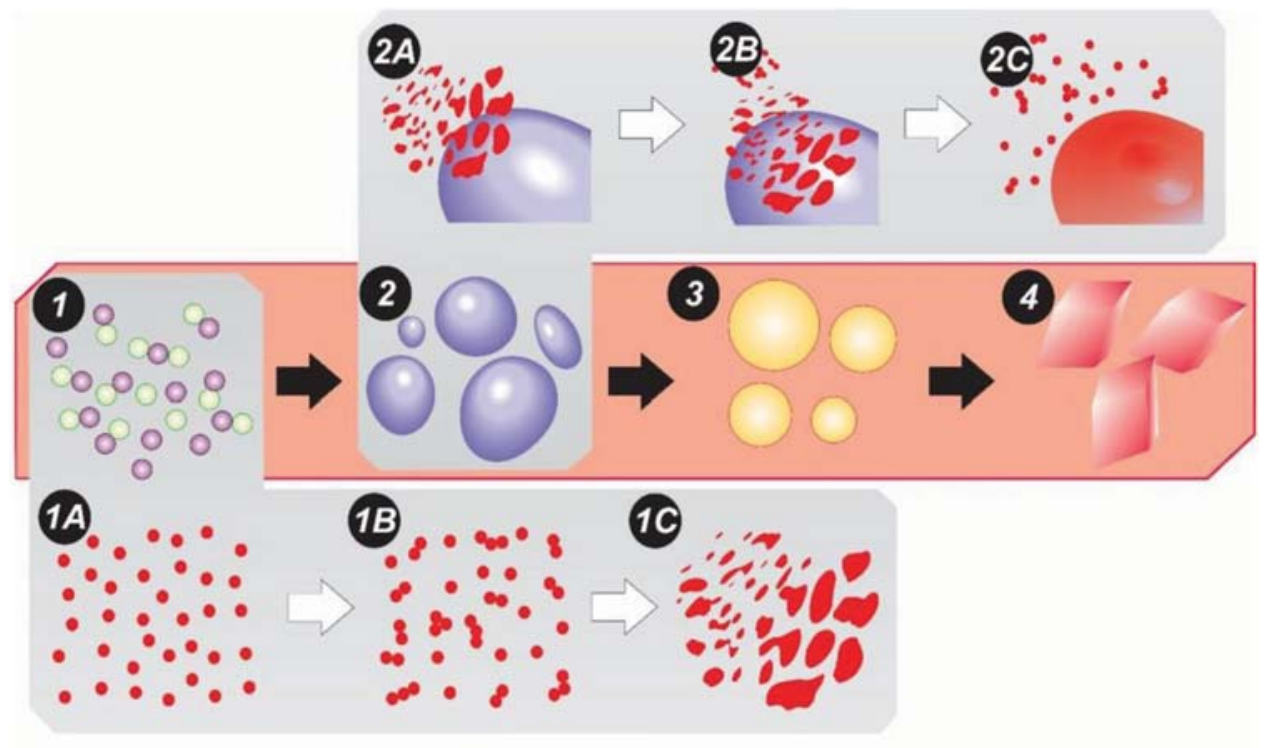

Figure 6. Schematic depiction of mineralization processes guided by unstructured proteins with low sequence complexity (red). The middle panel shows distinct inorganic forms associated with multistep nucleation and crystallization, viz., (1) ions and ion clusters, noncrystalline (2) liquid-like and (3) solid phases, and (4) crystals. In the prenucleation regime, free and bound ions induce structural transitions and self-association of biomolecules to rod- and sheet-like oligomers $(1 \mathrm{~A}-\mathrm{C})$. Subsequently, transient mineral phases formed via liquid-liquid demixing promote the accumulation of additives at the phase boundary of amorphous mineral droplets $(2 \mathrm{~A}, \mathrm{~B})$. Formation of vesicle walls confines the mineral phase, providing transient stabilizing against crystallization (2C).

suggest that although the investigated LC-IDRs can mediate vesicular structures, their impact on mineral formation can be fine-tuned by individual sequence-structure relations, post-translational modifications, ${ }^{[32,49]}$ as well as the selection of structural ensembles by inorganic interfaces and reaction conditions. This might elucidate the sequential interactions of ionic precursors with distinct regulatory proteins within vesicles during sea urchin skeletogenesis. ${ }^{[22]}$ Interestingly, the applied protein contents are in the range of those required for stabilization of LCPs by charged (bio)polymers such as poly(aspartic acid) and osteopontin, ${ }^{[50]}$ indicative that the contents of (bio)polymers in contact with the mineral precursors might be physiologically regulated. Overall, the progression of mineral nucleation and crystallization is chemically and physically programmed, encompassing not only the deposition of complex crystalline architectures, but also the controlled maturation of assimilated ion species toward LCPs and amorphous minerals. Since polymeric additives do not significantly affect the locus of the liquid-liquid binodal limit, ${ }^{[33]}$ the inhibition of mineral nucleation by LC-IDRs can be sufficient in prolonging the transient existence of (protein-containing) mineral-rich fluidic droplets and realizing the adsorption and organization of the structural subensemble of LC-IDPs at the phase boundary of mineral droplets, thus establishing the vesicle wall. In consequence, the shell structure might suppress a loss of mineralassociated hydration and biomolecules, ${ }^{[51]}$ restrict the degrees of freedom of the entrapped water molecules, ions, and polymers, ${ }^{[52]}$ as well as inhibit heterogeneous nucleation on the inner surfaces of the shells, hence engaging several physical parameters for the modulation of Ostwald ripening of the inorganic phase trapped in the water pools. ${ }^{[13 a, b]}$ This model is also consistent with previous in vivo studies which show that
SM50 participates in the initial stages of biomineralization, ${ }^{[21,53]}$ the application of antibodies against MSP130 inhibits spicule elongation, ${ }^{[53,54]}$ and for the prismatic layer, Prisilkin-39 is a negative regulator of crystallization. ${ }^{[23]}$

This proposed model can be further expanded in view of the heterogeneous biomolecular compositions of matrix vesicles. ${ }^{[55]}$ In environments wherein multiple macromolecules synergistically regulate mineralization, the competitive adsorption of nucleation and crystallization additives at interfaces of a maturating mineral phase (i.e., Vroman effect for liquid-liquid and liquid-solid interfaces) can play fundamental roles ${ }^{[56]}$ (Figure 7A). In this scenario, the binding and displacement of organic molecules from ions, ion clusters, droplets, and particles representative of the mineral phase are affected by (i) the molar mass of the additives and its affinity to a particular mineral phase, (ii) the changes in energy landscapes of conformational ensembles in response to ionic conditions and interfacial adsorption, and also (iii) the physicochemistry of the mineral-solvent interface in dependence of inorganic phase transitions (Figure 7B). Thus, the physical parameters associated with the "hard" and "soft" coronal constituents of inorganic droplets and particles can affect the structural features of minerals including morphology, hierarchical organization, and crystallography. Since minute contents of certain charged synthetic polymers can intrinsically stabilize fluidic mineral precursors, ${ }^{[50 a, 57]}$ the extrusion of protein units from a liquid condensed inorganic phase might partially contribute toward the formation of vesicle shells. Given that the mineralization additive based on the unstructured p53TAD domain does not significantly stabilize mineral precursors, the essential factors for protein-mediated mineral confinement are low sequence complexity as well as high self-association and restructuration 

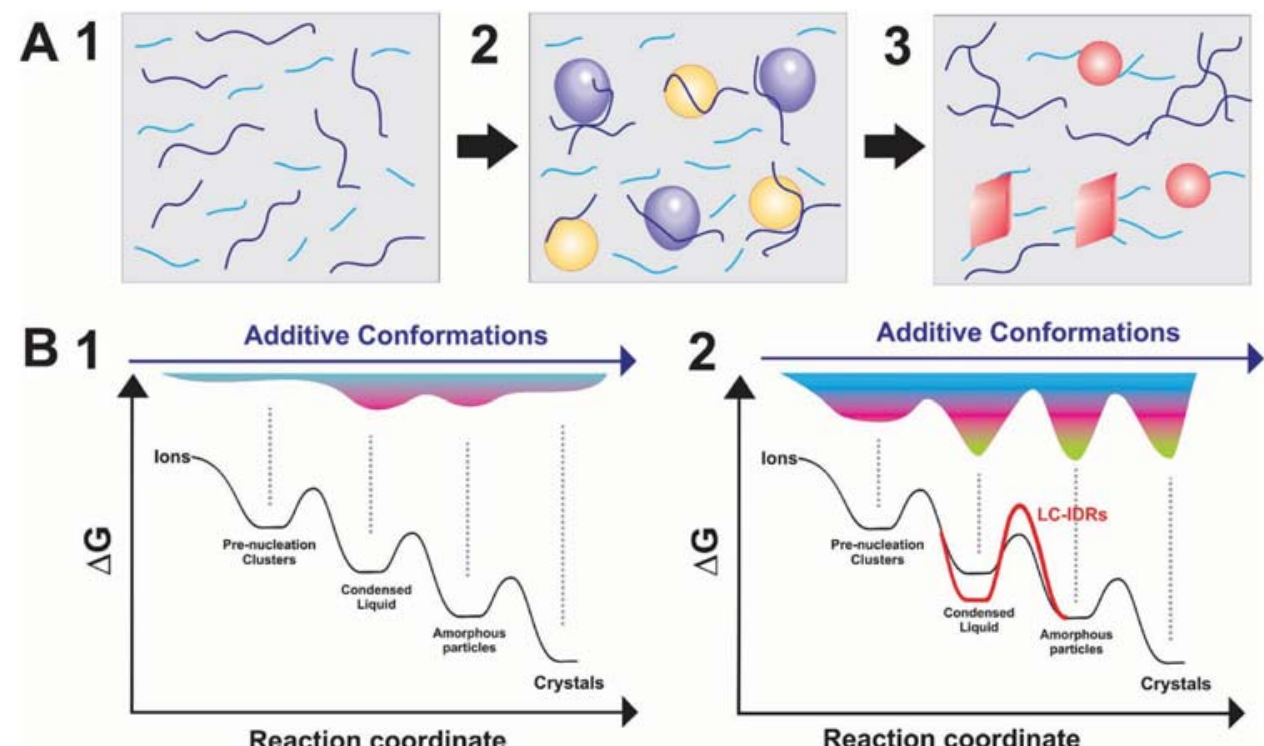

Figure 7. A) Dynamic Vroman effect for mineralization in heterogeneous environments. Hypothetical biomolecules (blue, cyan) exhibit distinct affinities toward (2) amorphous and (3) crystalline mineral surfaces, enabling distinct spatiotemporal distributions in relation to interfacial properties of the inorganic components. B) Changes in the energy landscape associated with additive conformations (top) for hypothetical disordered proteins "1" (left) and "2" (right) in context of the free energy profile of concurrent mineralization reactions from ions to crystals. With weak and nonspecific bioinorganic interactions, biopolymer "1" exhibits multiple local energy minima (pink) separated by small barriers, largely unaffected the inorganic phases. On the other hand, biopolymer "2" interacts specifically with discrete mineral species and presents corresponding local free energy minima (green). Based on this bioinorganic interplay, transitions toward amyloid-like $\beta$ conformations by the LC-IDRs enable a kinetic stabilization (and also possibly thermodynamic stabilization) of fluidic mineral precursors (red curve, right).

propensities of biomacromolecules in proximity of liquid-like mineral precursors. In this scenario, the enrichment of basic residues and the determinants of amyloid-like, elastomeric, or disordered propensities such as glycine and proline ${ }^{[58]}$ appear to collectively determine the synthetic replication of biomineralization-related confinement. In all, since IDP functions are based on an ensemble of structural states and the related transitions, ${ }^{[1 a, 59]}$ the effects of mesoscale properties including curvature, wettability, and charge as well as of molecular parameters such as crystallinity and hydration of mineral forms on the energy landscapes of disordered proteins will shed further light on the mechanistic roles of biopolymers in the formation of hard composites in nature (Figure 7B). For this, a conceptual framework for addressing the conformational states, folding/ unfolding kinetics, and aggregation of biomolecules in relation to nucleation and crystallization reactions of inorganic matter is required.

\section{Conclusion}

The chemical composition and structural design of biomineral-associated LC-IDPs appear fine-tuned for achieving an effective stabilization of amorphous minerals in aqueous environments. Since amorphous inorganic precursors effectively integrate with organic scaffolds and achieve complex crystalline architectures, the functional roles of biomacromolecules in course of mineral nucleation and transformation are closely linked to the ingrained physicochemical responsiveness of the biomolecules. Diverse inorganic precursors including ions, ion clusters, (liquid-like) amorphous phases, as well as crystalline forms elicit distinct effects on the conformational dynamism, self-association states, and phase behavior of biomolecules. This feature not only provides a window into the biophysical and molecular nature of material formation and growth, but also inspires the chemical programming of synthetic molecules toward inducible supramolecular assemblies, which in turn can achieve unprecedented spatiotemporal control over the fabrication of technological materials. In view of the sophisticated molecular responsiveness of biopolymers in nature's toolbox for constructing hard and soft materials, their potential in addressing primordial bioinorganic chemistry and in developing "smart" crystallization additives for biomedical purposes and crystal engineering is also promising.

\section{Experimental Section}

Recombinant Protein Production: Based on bioinformatics analysis, LC-IDR domains from SM50, LSM34, MSP130, and Prisilkin-39 were identified (Figure S2, Supporting Information). The TAD of p53 was selected as a reference additive. The domains were expressed intracellularly with a $\mathrm{N}$-terminal 6XHis-SUMO-tag in Escherichia coli BL27-CodonPlus-RIL strain (Agilent Technologies). ${ }^{[9]}$ Standard molecular biology procedures were followed. ${ }^{[60]}$ For protein expression, the induction of cell cultures was done using isopropyl- $\beta$-D-thiogalactopyranoside $\left(0.5 \times 10^{-3} \mathrm{M}\right)$ at $20^{\circ} \mathrm{C}$ for $16 \mathrm{~h}$. For the purification of the soluble SUMO-p53TAD, the debris-free cell lysate was loaded on a $\mathrm{Ni}^{2+}$-charged NTA agarose column. After initial washes with imidazole $\left(0.5 \times 10^{-3} \mathrm{M}\right)$, the protein of interest was eluted by using imidazole $\left(150 \times 10^{-3} \mathrm{M}\right)$. Final purification was performed by size exclusion chromatography 
using a Superdex 75 column (HiLoad 16/60, Amersham Biosciences) on an Akta FPLC system. Overexpression of the low complexity IDPs leads to an accumulation of the proteins in inclusion bodies. After cell lysis, the pellets were dispersed in PBS containing Triton $X(0.05 \%)$ and the mixtures were centrifuged at $30000 \mathrm{~g}$ for $20 \mathrm{~min}$. This procedure was repeated to remove residual soluble proteins from the pellets. The resulting pellets were dispersed in arginine $\left(200 \times 10^{-3} \mathrm{M}\right)$ solution, incubated at $4{ }^{\circ} \mathrm{C}$ and the soluble fractions were utilized for purification of His-tagged recombinant proteins by metal affinity chromatography (Figure S15, Supporting Information).

Quantitative Assessment of Mineral Nucleation: Under controlled parameters, mineral nucleation was performed using a commercia system from Metrohm (Filderstadt, Germany). The apparatus (Titrando 905) involves two dosing units controlled by a customized software (Tiamo v2.2). ${ }^{[25]}$ During the experiments, $\mathrm{CaCl}_{2}\left(10 \times 10^{-3} \mathrm{M}\right)$ was added

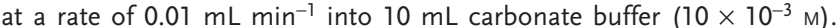
At the same time, the $\mathrm{pH}$ was kept constant at either 9.0 or 9.75 by the automatic counter-titration of $\mathrm{NaOH}$ solution $\left(10 \times 10^{-3} \mathrm{M}\right)$. The $\mathrm{pH}$ values and free $\mathrm{Ca}^{2+}$ concentrations were continuously monitored by a glass electrode (Metrohm, No. 6.0256.100) and a $\mathrm{Ca}^{2+}$ ion-selective electrode (ISE, Metrohm, No. 6.0508.110), respectively.

Structural Analyses: DLS was performed with a custom-built setup equipped with a goniometer with temperature control $(0.05 \mathrm{~K}$ step control), an attached single-photon detector ALV 6000/E, and a multiple tau digital correlator 5000/FAST from ALV (Langen, Germany). ${ }^{61]}$ For TEM, the solution was spotted on a formar coated $\mathrm{Cu}$ grid. Excess liquid was blotted and the grid was air-dried. Imaging was done by using a Libra120 (Zeiss Microscopy, Jena, Germany) TEM operated at $120 \mathrm{kV}$ with a beam current of $4 \mu \mathrm{A}$. Cryo-TEM was performed by blotting a drop onto a lacey carbon film covered $\mathrm{Cu}$ grid and vitrified by plunging into a liquid ethane bath in a temperature-controlled cryo-box (Zeiss Microscopy, Jena, Germany). The vitrified specimens were examined in a LEO EM922Omega EFTEM instrument (Zeiss Microscopy, Jena Germany). Images were recorded with a slow scan CCD camera (Ultrascan 1000, Gatan, Munich, Germany) using a software package (Digital Micrograph, Gatan, Munich, Germany). For FTIR spectroscopy, $\mathrm{CaCO}_{3}$ was precipitated by mixing equal volumes of $\mathrm{CaCl}_{2}\left(20 \times 10^{-3} \mathrm{M}\right)$ and carbonate buffer $\left(20 \times 10^{-3} \mathrm{M}, \mathrm{pH} 9\right)$ containing protein additives $(0.1 \mathrm{mg} \mathrm{mL}-1)$. After $20 \mathrm{~min}$ incubation at $25{ }^{\circ} \mathrm{C}$, the samples were centrifuged at $5000 \mathrm{~g}$ for $15 \mathrm{~min}$. FTIR analyses of the vacuum-dried pellets were done using a Perkin Elmer Spectrum 100 spectrometer in an attenuated total reflection (ATR) configuration. For elucidating protein structure, the mineralized samples were lyophilized and dispersed in $\mathrm{D}_{2} \mathrm{O}$. Protein pellets collected by centrifugation were measured for their IR spectra.

Congo Red and Thioflavin T (ThT) Assays: Stained protein samples were observed by using a Zeiss Axio Imager-M2m polarization microscope and a fluorescence microscope (Leica EC3 camera, LAS EZ software). Staining procedures were performed on wet samples. ${ }^{[62]}$ By following a standard protocol for the quantitation of $\beta$-sheet amyloid structures, ${ }^{[42]}$ samples with protein $(50 \mu \mathrm{g})$ and ThT $\left(10 \times 10^{-6} \mathrm{M}\right)$ in carbonate buffer $\left(10 \times 10^{-3} \mathrm{M}, \mathrm{pH} 9.0\right)$ were incubated in 96 -microwell plates at $25^{\circ} \mathrm{C}$ for $1 \mathrm{~h}$. In the presence of varying arginine contents, fluorescence intensities were detected using a microplate reader (Infinite M200, Tecan) with a $440 \mathrm{~nm}$ excitation and an emission scan of $460-529 \mathrm{~nm}$.

AUC: Sedimentation velocity experiments were performed in an XL-I AUC (Beckman Coulter) for investigating the solution properties of the fusion products. The moving boundary was monitored by radial scans at $280 \mathrm{~nm}$ with a UV absorption optical system. The data were analyzed to generate distributions of sedimentation coefficient and molar mass by using the SEDFIT program. ${ }^{[63]}$ Frictional coefficients $\left(f / f_{0}\right)$ were determined using 2DSA (Monte Carlo) performed with 20 iterations and grid resolution of 3600 by using Ultrascan III. [64]

Simulation of Mineralization-Induced Vesicles: The recombinant proteins were fluorescently labeled using sulforhodamine B sulfony chloride by following a standard protocol. ${ }^{[65]}$ After dialysis for the removal of excess dye, equal volumes of THF and aqueous protein solution were mixed and then analyzed by fluorescence microscopy.

\section{Acknowledgements}

A.R. acknowledges fellowships from the Konstanz Research Schoo Chemical Biology and Freiburg Institute for Advanced Studies. M.D. thanks the Bavarian Polymer Institute and the Collaborative Research Centre SFB840 of the German Research Foundation (DFG) for financial support. D.G. is a research fellow of the Zukunftskolleg of the University of Konstanz.

\section{Conflict of Interest}

The authors declare no conflict of interest.

\section{Keywords}

mineralization, nucleation, protein structures, self-assembly, vesicles

[1] a) V. N. Uversky, Int. J. Biochem. Cell Biol. 2011, 43, 1090 b) P. E. Wright, H. J. Dyson, Nat. Rev. Mol. Cell Biol. 2015, 16, 18.

[2] P. Romero, Z. Obradovic, X. Li, E. C. Garner, C. J. Brown, A. K. Dunker, Proteins: Struct., Funct., Bioinf. 2001, 42, 38.

[3] K. Sugase, H. J. Dyson, P. E. Wright, Nature 2007, 447, 1021.

[4] P. Tompa, C. Szasz, L. Buday, Trends Biochem. Sci. 2005, 30, 484.

[5] C. W. Pak, M. Kosno, A. S. Holehouse, S. B. Padrick, A. Mittal, R. Ali, A. A. Yunus, D. R. Liu, R. V. Pappu, M. K. Rosen, Mol. Cell 2016, 63, 72.

[6] F. Peysselon, B. Xue, V. N. Uversky, S. Ricard-Blum, Mol. BioSyst. 2011, 7, 3353

[7] K. Delak, C. Harcup, R. Lakshminarayanan, Z. Sun, Y. Fan, J. Moradian-Oldak, J. S. Evans, Biochemistry 2009, 48, 2272.

[8] a) D. Kurzbach, G. Platzer, T. C. Schwarz, M. A. Henen, R. Konrat, D. Hinderberger, Biochemistry 2013, 52, 5167; b) L. Fisher, D. Torchia, B. Fohr, M. Young, N. Fedarko, Biochem. Biophys. Res. Commun. 2001, 280, 460; c) A. L. Boskey, E. Villarreal-Ramirez, Matrix Biol. 2016, 52, 43.

[9] A. Rao, J. Seto, J. K. Berg, S. G. Kreft, M. Scheffner, H. Cölfen, J. Struct. Biol. 2013, 183, 205.

[10] L. Kalmar, D. Homola, G. Varga, P. Tompa, Bone 2012, 51, 528.

[11] T. M. Kapłon, G. Rymarczyk, M. Nocula-Ługowska, M. Jakób, M. Kochman, M. Lisowski, Z. Szewczuk, A. Ozyhar, Biomacromolecules 2008, 9, 2118.

[12] a) F. F. Amos, J. S. Evans, Biochemistry 2009, 48, 1332; b) Y. Lin, G. Jia, G. Xu, J. Su, L. Xie, X. Hu, R. Zhang, Acta Biochim. Biophys. Sin. 2014, 46, 709; c) M. Ndao, E. Keene, F. F. Amos, G. Rewari, C. B. Ponce, L. Estroff, J. S. Evans, Biomacromolecules 2010, 11, 2539; d) I. Perovic, E. P. Chang, M. Lui, A. Rao, H. Cölfen, J. S. Evans, Biochemistry 2014, 53, 2739.

[13] a) J.-M. Ha, J. H. Wolf, M. A. Hillmyer, M. D. Ward, J. Am. Chem. Soc. 2004, 126, 3382; b) C. J. Stephens, S. F. Ladden, F. C. Meldrum, H. K. Christenson, Adv. Funct. Mater. 2010, 20, 2108; c) A. Rao, H. Cölfen, J. Struct. Biol. 2016, 196, 232; d) T. M. Stawski, 
T. Roncal-Herrero, A. Fernandez-Martinez, A. Matamoros-Veloza, R. Kröger, L. G. Benning, Phys. Chem. Chem. Phys. 2018, 20, 13825.

[14] a) S. Y. Bahn, B. H. Jo, Y. S. Choi, H. J. Cha, Sci. Adv. 2017, 3, e1700765; b) I. Perovic, A. Davidyants, J. S. Evans, ACS Omega 2016, 1, 886; c) G. Jain, M. Pendola, Y.-C. Huang, J. J. Colas, D. Gebauer, S. Johnson, J. S. Evans, Biochemistry 2017, 56, 3607.

[15] a) B. L. Smith, T. E. Schäffer, M. Viani, J. B. Thompson, N. A. Frederick, J. Kindt, A. Belcher, G. D. Stucky, D. E. Morse, P. K. Hansma, Nature 1999, 399, 761; b) E. Weber, B. Pokroy, CrystEngComm 2015, 17, 5873.

[16] M. Wojtas, R. Hołubowicz, M. Poznar, M. Maciejewska, A. Ożyhar, P. Dobryszycki, Biochemistry 2015, 54, 6525.

[17] C. B. Ponce, J. S. Evans, Cryst. Growth Des. 2011, 11, 4690

[18] M. Pendola, J. S. Evans, J. Phys. Chem. B 2018, 122, 1161.

[19] a) A. H. Mao, S. L. Crick, A. Vitalis, C. L. Chicoine, R. V. Pappu, Proc. Natl. Acad. Sci. USA 2010, 107, 8183; b) S. Müller-Späth, A. Soranno, V. Hirschfeld, H. Hofmann, S. Rüegger, L. Reymond, D. Nettels, B. Schuler, Proc. Natl. Acad. Sci. USA 2010, 107, 14609.

[20] K. Mann, F. H. Wilt, A. J. Poustka, Proteome Sci. 2010, 8, 33.

[21] F. Wilt, L. Croker, C. E. Killian, K. McDonald, Invertebr. Biol. 2008, $127,452$.

[22] C. E. Killian, F. H. Wilt, Exp. Cell Res. 2017, 359, 205.

[23] Y. Kong, G. Jing, Z. Yan, C. Li, N. Gong, F. Zhu, D. Li, Y. Zhang, G. Zheng, H. Wang, J. Biol. Chem. 2009, 284, 10841.

[24] T. R. Butt, S. C. Edavettal, J. P. Hall, M. R. Mattern, Protein Expression Purif. 2005, 43, 1.

[25] a) D. Gebauer, A. Völkel, H. Cölfen, Science 2008, 322, 1819; b) D. Gebauer, H. Cölfen, A. Verch, M. Antonietti, Adv. Mater. 2009, $21,435$.

[26] V. N. Uversky, J. R. Gillespie, A. L. Fink, Proteins: Struct., Funct., Bioinf. 2000, 41, 415.

[27] J. J. Ward, L. J. McGuffin, K. Bryson, B. F. Buxton, D. T. Jones, Bioinformatics 2004, 20, 2138.

[28] Z. Dosztányi, V. Csizmok, P. Tompa, I. Simon, Bioinformatics 2005, 21, 3433.

[29] Z. R. Yang, R. Thomson, P. Mcneil, R. M. Esnouf, Bioinformatics 2005, 21, 3369.

[30] A. Rao, J. K. Berg, M. Kellermeier, D. Gebauer, Eur. J. Mineral. 2014, 26, 537.

[31] A. Picker, M. Kellermeier, J. Seto, D. Gebauer, H. Cölfen, Z. Kristallogr. - Cryst. Mater. 2012, 227, 744.

[32] E. P. Chang, I. Perovic, A. Rao, H. Cölfen, J. S. Evans, Biochemistry 2016, 55, 1024.

[33] F. Sebastiani, S. L. Wolf, B. Born, T. Q. Luong, H. Cölfen, D. Gebauer, M. Havenith, Angew. Chem., Int. Ed. 2017, 56, 490.

[34] A. Rao, H. Cölfen, Biophys. Rev. 2016, 1.

[35] a) L. B. Gower, Chem. Rev. 2008, 108, 4551; b) P. Kaempfe, V. R. Lauth, T. Halfer, L. Treccani, M. Maas, K. Rezwan, J. Am. Ceram. Soc. 2013, 96, 736.

[36] M. A. Bewernitz, D. Gebauer, J. Long, H. Cölfen, L. B. Gower, Faraday Discuss. 2012, 159, 291.

[37] a) E. M. Pouget, P. H. Bomans, J. A. Goos, P. M. Frederik, N. A. Sommerdijk, Science 2009, 323, 1455; b) E. DiMasi, T. Liu, M. J. Olszta, L. B. Gower, MRS Proc. 2005, 873, K10.6.
[38] N. Vidavsky, S. Addadi, J. Mahamid, E. Shimoni, D. Ben-Ezra, M. Shpigel, S. Weiner, L. Addadi, Proc. Natl. Acad. Sci. USA 2014, $111,39$.

[39] a) P. T. Yu, C. Tsao, C. C. Wang, C. Y. Chang, C. H. Wang, J. C. C. Chan, Angew. Chem., Int. Ed. 2017, 56, 16202; b) M. H. Nielsen, S. Aloni, J. J. De Yoreo, Science 2014, 345, 1158.

[40] Y. Politi, T. Arad, E. Klein, S. Weiner, L. Addadi, Science 2004, 306, 1161.

[41] G. Zandomeneghi, M. R. Krebs, M. G. McCammon, M. Fändrich, Protein Sci. 2004, 13, 3314.

[42] H. LeVine, Methods Enzymol. 1999, 309, 274.

[43] K. Tsumoto, M. Umetsu, I. Kumagai, D. Ejima, J. S. Philo, T. Arakawa, Biotechnol. Prog. 2004, 20, 1301.

[44] S. E. Wolf, J. Leiterer, V. Pipich, R. Barrea, F. Emmerling, W. Tremel, J. Am. Chem. Soc. 2011, 133, 12642.

[45] A. G. Checa, J. H. Cartwright, M.-G. Willinger, Proc. Natl. Acad. Sci. USA 2009, 106, 38.

[46] A. Akiva, G. Malkinson, A. Masic, M. Kerschnitzki, M. Bennet, P. Fratzl, L. Addadi, S. Weiner, K. Yaniv, Bone 2015, 75, 192.

[47] H. Anderson, Lab. Invest. 1989, 60, 320.

[48] S. Sviben, A. Gal, M. A. Hood, L. Bertinetti, Y. Politi, M. Bennet, P. Krishnamoorthy, A. Schertel, R. Wirth, A. Sorrentino, Nat. Commun. 2016, 7, 11228.

[49] G. Jain, M. Pendola, E. Koutsoumpeli, S. D. Johnson, J. S. Evans, Biochemistry 2018, 57, 3032.

[50] a) L. B. Gower, D. J. Odom, J. Cryst. Growth 2000, 210, 719; b) D. E. Rodriguez, T. Thula-Mata, E. J. Toro, Y.-W. Yeh, C. Holt, L. S. Holliday, L. B. Gower, Acta Biomater. 2014, 10, 494.

[51] S. J. Homeijer, R. A. Barrett, L. B. Gower, Cryst. Growth Des. 2010, 10, 1040.

[52] M.-C. Bellissent-Funel, Eur. Phys. J. E 2003, 12, 83.

[53] F. H. Wilt, Zool. Sci. 2002, 19, 253.

[54] M. C. Farach-Carson, D. D. Carson, J. L. Collier, W. J. Lennarz, H. R. Park, G. C. Wright, J. Cell Biol. 1989, 109, 1289.

[55] E. E. Golub, Biochim. Biophys. Acta, Gen. Subj. 2009, 1790, 1592.

[56] L. Vroman, A. L. Adams, Surf. Sci. 1969, 16, 438.

[57] B. Cantaert, Y. Y. Kim, H. Ludwig, F. Nudelman, N. A. Sommerdijk, F. C. Meldrum, Adv. Funct. Mater. 2012, 22, 907.

[58] S. Rauscher, S. Baud, M. Miao, F. W. Keeley, R. Pomes, Structure 2006, 14, 1667

[59] H. J. Dyson, P. E. Wright, Nat. Rev. Mol. Cell Biol. 2005, 6, 197.

[60] J. Sambrook, E. F. Fritch, T. Maniatis, Molecular Cloning: A Laboratory Manual, Cold Spring Harbor Laboratory Press, Cold Spring Harbor, NY 1989.

[61] H. Cölfen, L. Qi, Chem. - Eur. J. 2001, 7, 106.

[62] a) H. Puchtler, F. Sweat, M. Levine, J. Histochem. Cytochem. 1962, 10, 355; b) M. M. Picken, G. A. Herrera, Amploid and Related Disorders, Springer, Berlin 2012, p. 187.

[63] P. Schuck, Biophys. J. 2000, 78, 1606.

[64] E. Brookes, W. Cao, B. Demeler, Eur. Biophys. J. Biophys. Lett. 2010, 39, 405.

[65] G. T. Hermanson, Bioconjugate Techniques, Academic, San Diego, CA 2013. 